\title{
Exploring vasomotor mechanisms using PKG specific inhibitors Wolfgang Dostmann*
}

\author{
Address: University of Vermont, College of Medicine, Department of Pharmacology, Burlington, Vermont, USA \\ Email: Wolfgang Dostmann* - wolfgang.dostmann@uvm.edu \\ * Corresponding author
}

from 2nd International Conference of cGMP Generators, Effectors and Therapeutic Implications

Potsdam, Germany, 10-12 June, 2005

Published: 16 June 2005

BMC Pharmacology 2005, 5(SuppI I):SI5 doi:I0.1 I86/I47I-22I0-5-SI-SI5

The regulation of vascular tone is fundamentally linked to the activity of cGMP-dependent protein kinase (PKG). cGMP-induced activation of PKG presents a key step in the nitric oxide (NO) mediated reduction of intracellular $\mathrm{Ca}^{2+}$ and ultimately in vascular smooth muscle (VSM) relaxation. 'Acute' PKG inhibition in the vasculature by the use of our recently developed potent and membranepermeable PKG inhibitors, point to a novel, cGMP-independent role for PKG in smooth muscle. First, work done using DT-2 and the proteolytically stable analog (D)DT-2 suggests that constitutive PKG activity may play a functional role in the control of vascular tone. A second key discovery implies that the expression of PKG in small resistance arteries is dependent on intraluminal pressure. Virtually nothing is known about the role of basal PKG activity as well as the mechano-sensitive expression of PKG in the control of VSM relaxation. Both findings suggest a new concept, whereby PKG may play a role in modulating vascular tone autonomous of cGMP. 